\title{
Noncardiac Chest Pain in Young Patients: Author's Reply
}

TO THE EDITOR: The prevalence of noncardiac chest pain (NCCP) differs by the type of study performed: hospital-based studies, community-based studies or general practice studies. In the previous two studies, ${ }^{1,2}$ we reported that $38 \%-41 \%$ of NCCP in average aged subjects had gastroesophageal reflux disease (GERD) diagnosed by endoscopy and 24-hour esophageal $\mathrm{pH}$ monitoring, with similar protocol to this study on young aged NCCP. ${ }^{3}$ Comparing with our previous data, our present ratio of GERD-related NCCP (30\%) was low, although the young age is one of the potential risk factors for $\mathrm{NCCP}^{4}$ In this study, gastroenterologic causes such as peptic ulcer disease, gastrointestinal surgery and liver disease were excluded. Several pathophysiological mechanisms of NCCP have been suggested including GERD, esophageal motility disorders, visceral hyperalgesia and psychiatric disturbances and especially little are known about the characteristics of NCCP related to non GERD. Our data suggest that there may be more possibilities of other pathophysiological mechanisms except GERD in the young aged NCCP such as visceral hyperalgesia, psychiatric disturbances and so on. Therefore, as mentioned by the editor, management of the rest of the NCCP patients with non GERD is very important. The recent prospective case-control study by Lee et al $^{5}$ suggested a new serotonin-norepinephrine reuptake inhibitor to aid in establishing the strategy for management by showing significantly improved symptoms in $52 \%$ of young adult patients with functional chest pain. In this study, typical GERD symptoms were more frequent in NCCP patients with GERD than in NCCP patients with non GERD (78\% vs 29\%). Therefore, typical GERD symptoms as well as proton pump inhibitor test may be a fair predictor for GERD-related NCCP in young aged patients, despite the rela- tively low prevalence of GERD-related NCCP. In addition, the long-term natural history of NCCP is one of our major concerns, so we need to monitor these NCCP patients more thoroughly.

Tae Ho Seo, MD Jeong Hwan Kim, MD Department of Internal Medicine Digestive Disease Center Konkuk University School of Medicine Konkuk University Medical Center Seoul, Korea

1. Kim JH, Rhee PL, Park EH, Son HJ, Kim JJ, Rhee JC. Clinical usefulness of subgrouping of patients with non-cardiac chest pain according to characteristic symptoms in Korea. J Gastroenterol Hepatol 2007;22:320-325.

2. Kim JH, Sinn DH, Son HJ, Kim JJ, Rhee JC, Rhee PL. Comparison of one-week and two-week empirical trial with a high-dose rabeprazole in non-cardiac chest pain patients. J Gastroenterol Hepatol 2009;24:1504-1509.

3. Seo TH, Kim JH, Lee JH, et al. Clinical distinct features of noncardiac chest pain in young patients. J Neurogastroenterol Motil 2010;16:166-171.

4. Eslick GD. Noncardiac chest pain: epidemiology, natural history, health care seeking, and quality of life. Gastroenterol Clin North Am 2004;33:1-23.

5. Lee H, Kim JH, Son HJ, et al. Efficacy of venlafaxine for symptomatic relief in young adult patients with functional chest pain-A randomized, double-blind, placebo-controlled, crossover trial. Am J Gastroenterol 2010;105:1504-1512.

\section{Conflicts of interest: None.}

